\title{
AN EXAMPLE OF A BLOCH FUNCTION
}

\author{
RICHARD M. TIMONEY \\ Department of Mathematics \\ Indiana University \\ Bloomington, Indiana 47401 U.S.A.
}

(Received November 3, 1978)

ABSTRACT. A Bloch function is exhibited which has radial limits of modulus one almost everywhere but fails to belong to $\mathrm{H}^{\mathrm{P}}$, for each $0<\mathrm{p} \leq \infty$. KEY WORDS AND PHRASES. Bloch function.

AMS (MOS) SUBJECT CLASSIFICATION (1970) CODES. $30 A 78$

1. INTRODUCTION.

The purpose of this note is to give an example which seems to be useful in settling several questions about Bloch functions.

Let $\mathrm{E}$ be the subset of the complex plane $\mathbb{a}$ consisting of the closed unit disc together with the Gaussian integers $\mathbb{Z}^{2}$. Let $G$ be the complement of $E$ in in $\mathbb{C}$. Let $\mathrm{g}: \mathrm{D} \rightarrow \mathrm{G}$ be the analytic universal covering map of $\mathrm{G}$ given by the uniformization theorem ( $D$ denotes the unit disc).

PROPOSITION. The function $g$ is an unbounded Bloch function with the properties

(i) $g$ has a radial limit $g\left(e^{i \theta}\right)$ at almost every point $e^{i \theta}$ of the unit circle. 
(ii) the function $g\left(e^{i \theta}\right)$ is of modulus one almost everywhere on the unit circle,

(iii) $g$ is the reciprocal of a singular inner function, and so $g$ does not belong to any $\mathrm{H}^{\mathrm{p}}$ class.

Bloch functions on the unit disc may be defined as those analytic functions $f$ on $D$ for which the radii of the schlicht discs in the range of $f$ are bounded above. The Bloch functions are somewhat analagous to functions in the disc algebra--Bloch functions can be characterized (see [1]) as those analytic functions which are uniformly continuous when $\mathrm{D}$ is given the hyperbolic metric and $\mathbb{C}$ the Euclidean metric. Since Bloch functions may be characterized (see [1]) as those analytic functions $f$ on $D$ for which the quantity $\left|f^{\prime}(z)\right|\left(1-|z|^{2}\right)$ is bounded for $z \in D$, it follows that the modulus of a Bloch function grows rather slowly--at most as fast as $\log (1 /(1-|z|))$. Because functions in the disc algebra and bounded functions have good boundary behaviour, it is natural to ask about boundary values of Bloch functions-in particular about radial boundary values. (It is shown in [4] that a Bloch function has a radial limit at a point of the unit circle if and only if it has a non-tangential limit there.)

In [5], Pommerenke gave an example of a Bloch function with radial limits almost nowhere. The example given here is constructed in a similar way, but it contrasts with Pommerenke's in that it shows that Bloch functions which have radial limits almost everywhere need not be particularly we11-behaved.

The example answers a question posed by Joseph Cima (private communication). He asked whether a Bloch function which has radial limits 
almost everywhere and has the additional property that the boundary function belongs to $\mathrm{L}^{\mathrm{P}}$ need be in $\mathrm{H}^{\mathrm{P}}$. The function $\mathrm{g}$ provides a negative answer to this question since $g\left(e^{i \theta}\right) \in L^{\infty}$ while $g \notin H^{p}$ for any $0<\mathrm{p} \leq \infty$. In fact $\mathrm{g}$ does not belong to the class $\mathrm{N}^{+}$(see [2] p. 25) which contains $\mathrm{H}^{\mathrm{p}}$ for every $\mathrm{p}$.

PROOF. It is evident that $g$ is an unbounded Bloch function. Also, to verify properties (i), (ii) and (iii), it is clearly sufficient to verify (iii).

To establish (iii), consider the analytic function $f=1 / g$ on $D$. The function $f$ is bounded (by 1 ) and is the universal covering map : $D \rightarrow D-K$, where $K$ is the countable set

$$
\{0\} \cup\{1 /(m+i n)|m, n \in \mathbb{Z},| m+i n \mid>1\} .
$$

Being a bounded analytic function, $f$ has radial limits almost everywhere on the unit circle. It is easy to see from the properties of covering maps that these radial limits are either of modulus 1 or else belong to $K$. To complete the proof that $f$ is a singular inner function, it is only necessary to show that the radial limit $f\left(e^{i \theta}\right)$ belongs to $K$ on a subset of the unit circle of measure zero.

But, for each $k \in K$ it is true that the set of $e^{i \in}$ for which $f\left(e^{i \theta}\right)=k$ has measure zero (see [2] p. 17). Since $K$ is countable, it follows that the set of $e^{i \theta}$ for which $f\left(e^{i \theta}\right)$ belongs to $k$ also has measure zero. The proof is now complete.

The example may also be viewed as elucidating the almost total lack of relationships between the class $B$ of $B l o c h$ functions on $D$ and the subclasses $\mathrm{H}^{\mathrm{P}}$ and $\mathrm{N}^{+}$of the Nevanlinna class $\mathrm{N}$ (see [2]). The only 
containment which holds between $B$ and the other classes is the relation $\mathrm{H}^{\infty} \subseteq B$. It is known that $\mathrm{H}^{\mathrm{P}} \nsubseteq B$ for any $0<\mathrm{p}<\infty$ and that $B \nsubseteq \mathrm{N}$. The example $g$ given above belongs to $B \cap \mathrm{N}$ but not to $\mathrm{N}^{+}$. The fact $B \nsubseteq \mathrm{N}$ is shown by the example of Pommerenke's [5] mentioned above. Finally, the example given here can be modified to show that there is no $\delta>0$ such that an analytic function $f: D \rightarrow \mathbb{C}$ satisfying

$$
f\left(e^{i \theta}\right)=\operatorname{Lim}_{r \rightarrow 1} f\left(r e^{i \theta}\right)=1
$$

almost everywhere on the unit circle must have a disc of radius $\delta$ in its range. (Merely replace $\mathbb{Z}^{2}$ by $\delta \mathbb{Z}^{2}$ in the construction of $g$ ). This answers a question raised by J.S. Hwang. By contrast, he showed (see [3]) that a singular inner function (for example) must have a (Schlicht) disc of radius at least $2 \mathrm{~B} / \mathrm{e}$ in its range, where $\mathrm{B}$ denotes Bloch's constant.

\section{REFERENCES}

1. Anderson, J.M., J.G. Clunie, Ch. Pommerenke. On Bloch Functions and Normal Functions, J. Reine Angew. Math. 270(1974) 12-37.

2. Duren, P. $\underline{\mathrm{H}}^{\mathrm{P}}$ Spaces, Academic Press, 1970.

3. Hwang, J.S., On an Extremal Property of Doob's Class (preprint, 1978).

4. Lehto, 0., K.I. Virtanen. Boundary behaviour and normal meromorphic functions. Acta Math. 97(1957) 47-65.

5. Ch. Pommerenke. On Bloch functions. J. London Math. Soc. (2) 2(1970) 689-695. 


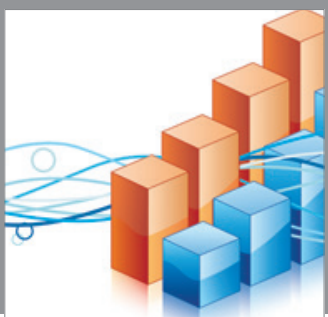

Advances in

Operations Research

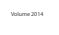

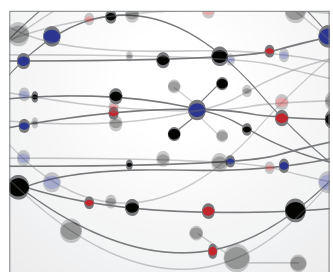

\section{The Scientific} World Journal
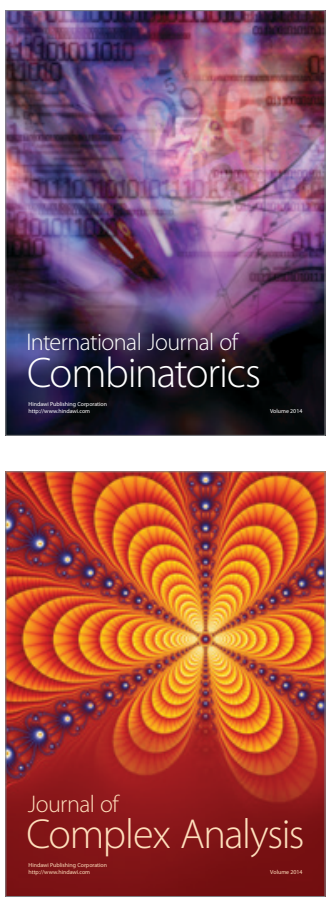

International Journal of

Mathematics and

Mathematical

Sciences
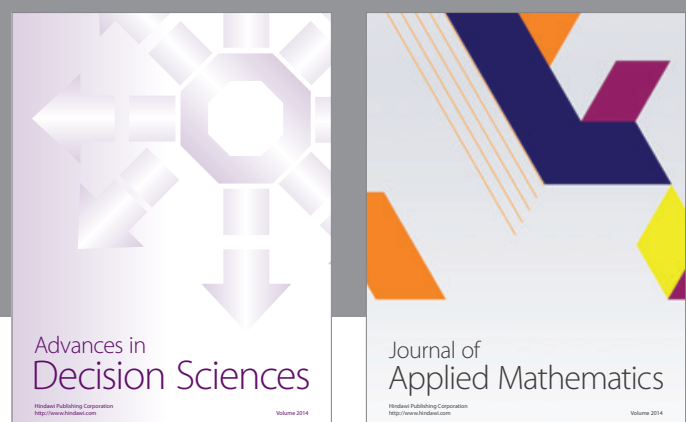

Journal of

Applied Mathematics
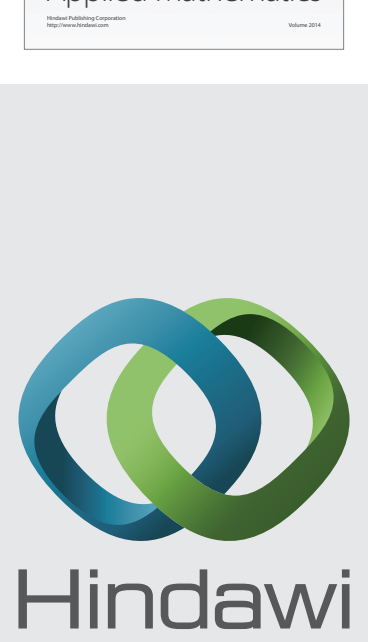

Submit your manuscripts at http://www.hindawi.com
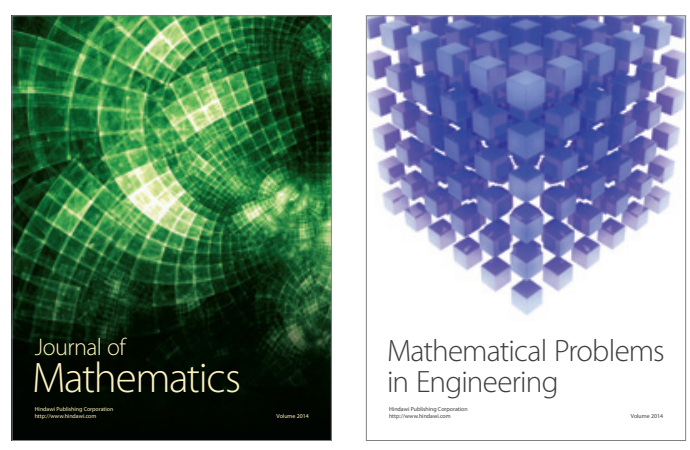

Mathematical Problems in Engineering
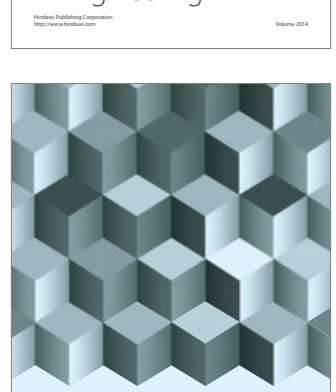

Journal of

Function Spaces
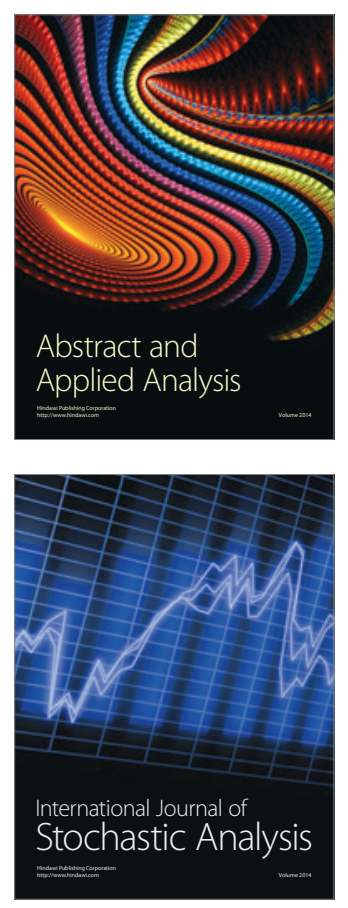

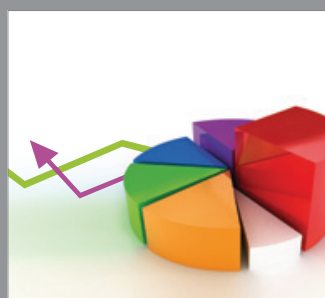

ournal of

Probability and Statistics

Promensencen
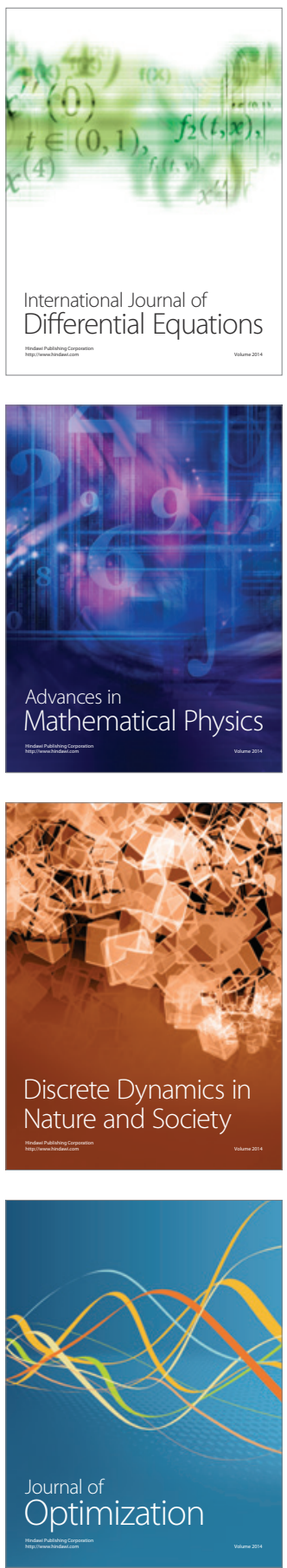